\title{
Editorial
}

\section{Bcl-G acquitted of murder!}

\author{
D Tischner ${ }^{1}$ and A Villunger ${ }^{*, 1}$ \\ Cell Death and Disease (2012) 3, e405; doi:10.1038/cddis.2012.147; published online 11 October 2012
}

Proteins of the $\mathrm{Bcl}-2$ family are characterized by the presence of structural motives, referred to as Bcl-2 homology $(\mathrm{BH})$ domains that orchestrate protein-protein interactions within the family. The prosurvival members, such as $\mathrm{Bcl}-2$ or $\mathrm{Bcl}-\mathrm{xL}$, usually contain four such domains $(\mathrm{BH} 1-4)$, while their proapoptotic opponents, Bax/Bak-like proteins and the BH3-only proteins, contain three or only one such domain, that is, the $\mathrm{BH} 1,2$ and 3 or the $\mathrm{BH} 3$ domain, respectively. ${ }^{1}$ In concert, these proteins regulate cell death by the mitochondrial apoptosis pathway. However, some members of the family cannot be fully integrated in one of the three described subgroups and their specific contribution to apoptosis regulation remains elusive. One such candidate of an 'orphan' member is Bcl2L14, also called Bcl-G (Bcl-Gonad). ${ }^{2}$

$\mathrm{Bcl}-\mathrm{G}$ is evolutionary conserved in mammals, reptiles and birds and in humans the locus encodes for a long and a short isoform, $B c l-G_{L}$ and $B c l-G_{S}$, that arise due to differential splicing that changes the distal reading frame in exon 5, creating a premature STOP codon. ${ }^{2}$ Whereas $B c l-G_{L}$ reportedly displays wide tissue expression, $\mathrm{Bcl}-\mathrm{G}_{\mathrm{S}}$ was only detected in testis by RT-PCR analysis. The alternative amino acid sequences in the respective carboxy-termini of both isoforms also confer differences in their cellular distribution. Whereas epitope-tagged $\mathrm{Bcl}-\mathrm{G}_{\mathrm{L}}$ was found dispersed diffusely throughout the cytoplasm, Bcl- $\mathrm{G}_{S}$ localized to cytosolic organelles. Of note, $\mathrm{Bcl}-\mathrm{G}_{\mathrm{S}}$ contains only a $\mathrm{BH} 3$ domain, reportedly induces apoptosis upon overexpression and interacts with $\mathrm{Bcl}-\mathrm{xL}$ and $\mathrm{Bcl}-2$. Contrary to $\mathrm{Bcl}-\mathrm{G}_{\mathrm{S}}, \mathrm{Bcl}-\mathrm{G}_{\mathrm{L}}$ possesses also a $\mathrm{BH} 2$ domain but does not display any significant binding to $\mathrm{Bcl}-\mathrm{xL}$, nor apoptotic activity. Only after deletion of the $\mathrm{BH} 2$ domain $\mathrm{Bcl}-\mathrm{G}_{\mathrm{L}}$ induced cell death in vitro, comparable to $\mathrm{Bcl}-\mathrm{G}_{\mathrm{S}}$ overexpression. Of note, $\mathrm{Bcl}-\mathrm{G}$ is located on human chromosome 12p12, harboring at least one putative tumor suppressor and its expression was found reduced in human prostate and breast cancer samples, suggesting a function as anti-oncogene., ${ }^{3,4}$ However, sequence analysis of the $\mathrm{BH} 3$ domain of $\mathrm{Bcl}-\mathrm{G}$ in urinary bladder cancer and laryngeal squamous cell carcinomas failed to detect any mutations, suggesting that a possible tumor suppressive role may be unrelated to possible $\mathrm{Bcl}-2$ family interactions, ${ }^{5,6}$ and the analysis of the remaining allele of Bcl-G in pre-B childhood acute lymphoblastic leukemia patients that carry a $12 p$ hemizygous deletion also failed to reveal any loss-of-function, ${ }^{7}$ questioning a role as tumor suppressor.
Philippe Bouillet and colleagues ${ }^{8}$ started a whole-hearted effort to elucidate the protein expression pattern and physiological function of Bcl-G by the generation of a set of highly specific monoclonal antibodies and Bcl-G-deficient mice. ${ }^{9}$ Contrary to some commercially available antibodies that picked up a 22-kDa band by western blotting, the newly generated monoclonals detected only a single 38-kDa band that was not present in protein extracts from cells and tissues derived from Bcl-G-deficient mice. Using four different antibodies, they could confirm that in mice only one Bcl-G isoform exists, reflecting human $\mathrm{Bcl}-\mathrm{G}_{\mathrm{L}}$. Mouse $\mathrm{Bcl}-\mathrm{G}$ displays high expression in a range of epithelial and dendritic cells (DC) and is widely distributed among tissues. Yet, Bcl-G-deficient mice develop normally and display no obvious phenotype or signs of tissue hyperplasia, as one may expect upon loss of a pro-apoptotic gene. Although Bcl-G is highly expressed in spermatids and also in the epithelia of the mammary gland, Bcl-G-deficient mice show normal reproductive behavior. Interestingly, Bcl-G seems to share features with Bfk (Bcl-2 family kin), another orphan that contains only a $\mathrm{BH} 2$ and a $\mathrm{BH} 3$ domain with poor pro-apoptotic potential. Bfk was found highly regulated in its expression in the mammary gland, ${ }^{10}$ as well as in principal cells of the epididymis ${ }^{11}$ and the gastrointestinal tract. ${ }^{12}$

Bcl-G seems not required for intestinal epithelial cell differentiation and its loss had no impact on the subset composition of the hematopoietic system. Of note, whereas no significant protein levels were detected in lymphocytes, high expression was observed in $\mathrm{CD}^{+}$conventional DCs (cDCs), while plasmacytoid DCs lacked Bcl-G. Interestingly, $\mathrm{Bcl}-\mathrm{G}$ was upregulated in $\mathrm{cDCs}$ upon $\mathrm{CpG}$ oligonucleotide stimulation, suggesting a putative involvement in TLR-9mediated signaling pathways and innate immunity. Similar to other tissue and cell subtypes Bcl-G was not required for DC development and function, as monitored in antigen-cross presentation and in naive $\mathrm{T}$ cell activation assays.

Although in all former studies published on Bcl-G a proapoptotic function was reported, Bcl-G deficiency did not improve survival of DCs ex vivo. In accordance with this, Bouillet and Giam $^{9}$ confirmed that Bcl-G binds only weakly to antiapoptotic $\mathrm{Bcl}-2$ protein family members but, in contrast to the initial report, ${ }^{2}$ this interaction did not depend on a functional BH3-domain. Even more striking, grafting the $\mathrm{BH} 3-d o m a i n$ of $\mathrm{Bcl}-\mathrm{G}$ into pro-apoptotic Bim $\mathrm{S}$, ablated its ability to bind $\mathrm{Bcl}-\mathrm{xL}$ and to act pro-apoptotic, demonstrating

\footnotetext{
${ }^{1}$ Division of Developmental Immunology, BIOCENTER, Medical University Innsbruck, Innsbruck, Austria

*Corresponding author: A Villunger, Division of Developmental Immunology, Biocenter, Medical University Innsbruck, A-6020, Innsbruck, Austria. Tel: +43 5129003 70380; Fax: +43 5129003 73960; E-mail: Andreas.villunger@i-med.ac.at
} 
that whatever residual killing potential $\mathrm{Bcl}-\mathrm{G}$ might have, it does not involve classical Bcl-2 family interactions.

In search for additional clues, the authors exploited their newly generated monoclonal antibodies to immunopurify endogenous $\mathrm{Bcl}-\mathrm{G}$ from gastrointestinal epithelial cells, followed by mass spectrometry-based analysis. In direct comparison with lysates from $\mathrm{Bcl}-\mathrm{G}^{-/-}$mice, this led to the identification of 19 proteins that were found to co-purify with both antibodies, but none of them belonged to the Bcl-2 family. Intriguingly, in a yeast two-hybrid approach, the authors further identified the Transport particle protein (TRAPP) complex 6b (Trappc6b) as specific binding partner of Bcl-G. This finding is of interest, as also the MS-analysis revealed other components of the TRAPP complex (Trappc3, 4 and 5) as putative interactors, proteins highly conserved from yeast to man involved in vesicular transport in the early secretory pathway between the ER and Golgi apparatus. ${ }^{13}$ Thus, contrary to former reports where Bcl-G was described as a novel pro-apoptotic Bcl-2 family member the current data suggest that $\mathrm{Bcl}-\mathrm{G}$ is rather involved in vesicle trafficking and protein transport processes inside the cell.

The generation of specific monoclonal antibodies and BclG-deficient mice represent a major advance providing the most critical tools for further characterization of the physiological function of Bcl-G. Somewhat unfortunately, none of the antibodies recognizes human Bcl-G. Although Bcl-Gdeficient mice develop normal and display no obvious phenotype under steady state conditions, the function of Bcl-G under pro-inflammatory conditions and in tumor development remains to be explored in appropriate model systems. Especially, the putative role of Bcl-G in the early secretory pathway is intriguing, given its prominent expression in epithelial cells.

Despite the negative results in antigen-cross presentation studies, Bcl-G could still be required for the establishment of efficient immune responses in response to certain types of viruses. Also, DCs in the thymic medulla, known to present peripheral self-antigens to developing $T$ cells, express significant amounts of Bcl-G, raising the question whether it may be required for establishing central tolerance. Long-term follow-up and challenge of $\mathrm{BCl}-\mathrm{G}^{-/-}$mice in models of induced autoimmunity will help to address these possibilities. Along similar lines, a possible involvement of $\mathrm{Bcl}-\mathrm{G}$ in sensing or transmitting danger signals in innate immune cells, such as the one elicited by foreign DNA sequences after endocytosis via TLR-9 from endosomes, might be considered. Certainly, the former assumption that Bcl-G acts as a tumor suppressor by acting as a killer has to be revisited. As for now, Bcl-G is clearly acquitted of murder.

\section{Conflict of Interest}

The authors declare no conflict of interest.

1. Labi V et al. Cell Death Diff 2006; 13: 1325-1338.

2. Guo B, Godzik A, Reed JC. J Biol Chem 2001; 276: 2780-2785.

3. Latil A et al. Clin Cancer Res 2003; 9: 5477-5485.

4. Lin ML et al. Breast Cancer Res 2007; 9: R17.

5. Yoo NJ et al. Tumori 2007; 93: 281-283.

6. Soung YH et al. Pathology 2006; 38: 33-34.

7. Montpetit A et al. Leukemia 2004; 18: 1499-1504.

8. Giam M et al. Cell Death Dis 2012; 3: e378.

9. Bouillet P, Giam M. Cell Death Dis 2012; 3: e404.

10. Coultas $L$ et al. Cell Death Diff 2003; 10: 185-192.

11. Pujianto DA et al. Endocrinology 2007; 148: 3196-3204.

12. Dempsey CE et al. FEBS Lett 2005; 579: 3646-3650.

13. Yu S, Liang Y. Cell Mol Life Sciences 2012; e-pub ahead of print 6 June 2012.

(c) Cell Death and Disease is an open-access journal SOMERाIHISARESERVED published by Nature Publishing Group. This work is
licensed under the Creative Commons Attribution-NonCommercial-No Derivative Works 3.0 Unported License. To view a copy of this license, visit http://creativecommons.org/licenses/by-nc-nd/3.0/ 